\title{
Little research on effective tools to improve patient safety in the dental setting
}

\author{
Abstracted from \\ Bailey E, Tickle M, Campbell S, O'Malley L. \\ Systematic review of patient safety interventions in dentistry. \\ BMC Oral Health 2015; 15: 152. \\ Address for correspondence: E Bailey, NIHR Greater Manchester Primary Care Patient Safety \\ Translational Research Centre, Institute of Population Health - Centre for Primary Care, \\ University of Manchester, Manchester, UK. E-mail Edmund.bailey@manchester.ac.uk; edmund.bailey@uclh.nhs.uk
}

\section{Question: What tools are available to help improve patient safety in the dental setting?}

Data sources Medline via OVID, Embase via OVID, HMIC via OVID, CINAHL via EBSCO and Web of Science.

Study selection Descriptive, observational and experimental studies that used or described the development of patient safety interventions relating to dental care. Outcomes of interest were: patient safety, harm prevention, risk minimisation, patient satisfaction and patient acceptability, professional acceptability, efficacy, cost-effectiveness and efficiency.

Data extraction and synthesis All titles and abstracts were screened by at least two authors. The eligible studies were data extracted by two authors, with disagreements resolved by a third reviewer if necessary. A narrative approach was taken and quality assessed using CASP tools.

Results Nine studies were identified. Four described the use of checklists, three the use of reporting systems, one the use of electronic reminders and one the use of trigger tools. The risk of bias in the studies was high.

Conclusions The available literature on patient safety is in its infancy. Surgical checklists may be effective in reducing surgical errors.

\section{Commentary}

This systematic review is an important contribution to the improvement of safety in dental practice. It shows how little is known about both the incidence of safety-breaches and the interventions that may reduce these. This is, perhaps, not so surprising. Until the 1990s healthcare researchers generally paid very little attention to errors in care. ${ }^{1}$

Safety has been defined in different ways. The WHO defines it as 'the prevention of errors and adverse effects to patients associated with health care'. ${ }^{2}$ Another definition is that it is 'the avoidance, prevention and amelioration of adverse outcomes or injuries stemming from the process of healthcare', ${ }^{1}$ drawing attention to the outcome rather than the process and of the potential for patients to suffer physical or psychological harm from sources other than error.

Like much in the quality improvement (QI) world, interventions to improve patient safety are inevitably messy and complex, dependent on the local physical, psychological and social contexts that people work in. Thus, even if an intervention were to be shown to work well in one setting to improve patient safety, it may not work in another. That is not to suggest that there aren't tools that might be of help and that might be incorporated into practice routines, for example, as part of a plan-do-study-act (PDSA) or regular audit cycle. Certainly there are a large number of organisations promoting the uptake of interventions to improve patient safety, ${ }^{2-4}$ and for good reason: wrong teeth get extracted, ${ }^{5}$ patients swallow or inhale things that could cause them harm in the dental chair ${ }^{6}$ and patients die of anaphylaxis in reaction to commonly-used agents. ${ }^{7}$ In addition to errors around treatment, safety may be compromised by errors around diagnosis. ${ }^{8}$

This review sought to identify and assess tools that had been used to improve safety in the dental setting. It included both observational and experimental study types, which is fitting to a scoping-type review where it is unknown what work has been done in the field. ${ }^{9}$ They focused on dental practice in its broadest interpretation so as not to limit the potential to identify usable studies. They did restrict to English only, which may have meant potentially eligible research was excluded but the search was otherwise comprehensive. I think we can be fairly confident that most of what has been written on the subject of tools to improve safety in dentistry is here. However, there are other ways potentially to improve safety than tools, for example, through educational interventions. ${ }^{10}$

The review usefully separates those studies that were used to identify adverse events from those used to 'prevent, minimise or reduce adverse events'. This reflects opinion from elsewhere that 
simply having reporting systems, whilst an important component of a safety system, is not sufficient to improve or measure safety. ${ }^{11}$ Indeed, the safety reporting system in one large hospital in England only identified $5 \%$ of incidents that an analysis of the patients' documents identified. ${ }^{12}$

Several tools were identified but it is difficult to argue that any of them were effective in improving safety owing to the lack of controls in most studies. Checklists, reporting systems, trigger tools ('easily detectable, focused item in a patient's case notes that can help to lead to the identification of an adverse event') and alerts on electronic notes are described. Only the checklists were used in a way to prevent, minimise or reduce adverse events. The others could not possibly be a stand-alone intervention to improve safety.

It is interesting to note that another systematic review, on interventions to avoid wrong-site surgery, ${ }^{13}$ was only able to identify two randomised controlled trials from all of the surgical literature. One of those was the educational intervention cited earlier to reduce wrong-site tooth extractions. More generally, though, a review of 33 studies on the use of surgical checklists found them to be effective in improving surgical safety. ${ }^{14}$

In reality, as individuals engaged in improving the safety of care for our patients, we are likely to need to be aware of potential tools we can use. I think it will be the case for some time that we will not have robust randomised controlled trials to demonstrate the effectiveness of one or other tool. Given the importance of patient safety, we should not be put off by the absence of high quality research evidence but, rather, use QI approaches such as PDSA to try out in small ways some of the available tools to see what works in our particular contexts.

To increase our ability to improve safety, though, it would be helpful to be conscious of the other phenomena that are important in helping improving safety. For example, the safety culture of the organisation where we work, the resources available to us, organisational leadership and the need to anticipate future incidents. We can learn much about bringing about positive change by being aware of the broader QI literature, which recognises the multidimensional, flexible approaches needed. ${ }^{15}$

Dominic Hurst

Dental Public Health Unit, Barts and The London School of Medicine and Dentistry, Queen Mary University of London, London, UK and Department of Primary Care Health Sciences, University of Oxford, Oxford, UK.

1. Vincent CD. Patient safety. 2nd edn. Oxford: Wiley-Blackwell; 2010.

2. World Health Organisation. Patient Safety 2016 [cited 2016 28/05/2016]. Available from: http://www.euro.who.int/en/health-topics/Health-systems/patient-safety [Accessed 2 June 2016]

3. NHS Improving Quality. 2016 [cited 2016 28/05/2016]. Available from: http://www. nhsiq.nhs.uk/ [Accessed 2 June 2016]

4. Insitute for Healthcare Improvement. 2016 [cited 2016 28/05/2016]. Available from: http://www.ihi.org/ [Accessed 2 June 2016]

5. Saksena A, Pemberton MN, Shaw A, Dickson S, Ashley MP. Preventing wrong tooth extraction: experience in development and implementation of an outpatient safety checklist. Br Dent J 2014; 217: 357-362.

6. Atherton GJ, McCaul JA, Williams SA. Medical emergencies in general dental practice in Great Britain. Part 1: Their prevalence over a 10-year period. Br Dent / 1999; 186: 72-79.

7. Pemberton MN, Gibson J. Chlorhexidine and hypersensitivity reactions in dentistry. Br Dent / 2012; 213: 547-550.

8. McDonald KM, Matesic B, Contopoulos-loannidis DG, et al. Patient safety practices targeted at diagnostic errors: a systematic review. Ann Intern Med 2013; 158: 381 389.

9. Arksey H, O'Malley L. Scoping studies: towards a methodological framework. Int / Soc Res Methodol 2005; 8: 19-32.

10. Chang HH, Lee JJ, Cheng SJ, et al. Effectiveness of an educational program in reducing the incidence of wrong-site tooth extraction. Oral Surg Oral Med Oral Pathol Oral Radiol Endod 2004; 98: 288-294.

11. Vincent C, Aylin P, Franklin BD, et al. Is health care getting safer? Br Med J 2008; 337: a2426. doi: 10.1136/bmj.a2426.

12. Sari AB, Sheldon TA, Cracknell A, Turnbull A. Sensitivity of routine system for reporting patient safety incidents in an NHS hospital: retrospective patient case note review. Br Med J 2007; 334: 79.

13. Algie CM, Mahar RK, Wasiak J, Batty L, Gruen RL, Mahar PD. Interventions for reducing wrong-site surgery and invasive clinical procedures. Cochrane Database Syst Rev 2015; 3: CD009404. doi: 10.1002/14651858.CD009404.pub3.

14. Treadwell JR, Lucas S, Tsou AY. Surgical checklists: a systematic review of impacts and implementation. BMJ Qual Saf 2014; 23: 299-318. bmjqs-2012-001797.

15. Gabbay J, Le May A, Connell C, Klein J. Skilled for improvement? London: The Health Foundation; 2014. 81 p.

Evidence-Based Dentistry (2017) 17, 38-39. doi:10.1038/sj.ebd.6401163 